\title{
The impact of obesity on seasonal influenza: a single-center, retrospective study conducted in Israel
}

\author{
Alaa Atamna ${ }^{1,2}\left(\right.$ D $\cdot$ Ron Daskal $^{2} \cdot$ Tanya Babich $^{2,3} \cdot$ Gida Ayada $^{4} \cdot$ Haim Ben-Zvi ${ }^{5} \cdot$ Avishay Elis $^{2,4} \cdot$ Jihad Bishara $^{1,2}$. \\ Tomer Avni ${ }^{2,6}$
}

Received: 23 October 2020 / Accepted: 24 January 2021 / Published online: 11 February 2021

(C) The Author(s), under exclusive licence to Springer-Verlag GmbH, DE part of Springer Nature 2021

\begin{abstract}
Obesity is associated with an increased susceptibility to infections. Several studies have reported adverse clinical outcomes of influenza among obese individuals. Our aim was to examine the association between obesity and the clinical outcomes of hospitalized adult patients ill with seasonal influenza. Consecutive hospitalized adult patients between 10/2017 and 4/2018 with laboratory confirmed influenza A and B were divided into an obese group (body mass index (BMI) $\geq 30 \mathrm{~kg} / \mathrm{m}^{2}$ ) and controls. The primary outcome was a composite endpoint of 30-day all-cause mortality, vasopressor use, mechanical ventilation, ICU admission, and severe influenza complication (myocarditis and encephalitis). Secondary outcomes encompassed all the components of the primary outcome, 90-day all-cause mortality, occurrence of pneumonia, length of hospital stay, and 90-day readmission rates. The study comprised 512 hospitalized adults diagnosed with laboratory-confirmed influenza A (195/512) and B (317/512). Within this group, $17 \%$ (86/512) were classified obese; the remaining 83\% (426/512) were controls. Results of the composite outcome $(7 / 85,8 \%$ vs. $45 / 422,11 \% ; p=0.5)$ and the crude 30 -day all-cause mortality rate $(5 / 86,6 \%$ vs. $34 / 426,8 \%, p=0.5)$ were similar between the two groups. The multivariate analysis demonstrated that obesity was not a significant risk factor for influenza adverse events $(\mathrm{OR}=1.3$, CI 95\% 0.3-3.3; $p=0.5)$, whereas advanced age, chronic kidney disease, and hypoalbuminemia were significant risk factors $(\mathrm{OR}=1.03, \mathrm{OR}=2.7$, and $\mathrm{OR}=5.4$, respectively). Obesity was not associated with influenza-related morbidity and mortality among the hospitalized adults during the 2017-2018 influenza season. Further studies researching different influenza seasons are essential.
\end{abstract}

Keywords Influenza $\cdot$ Obesity $\cdot$ Infection $\cdot$ Mortality $\cdot$ BMI

This work was performed in partial fulfillment of the M.D. thesis requirement of the Sackler Faculty of Medicine, Tel Aviv University.

Alaa Atamna

a.atamna86@gmail.com

1 Infectious Disease Unit, Rabin Medical Center, Beilinson Hospital, Petach-Tikva, Israel

2 Sackler Faculty of Medicine, Tel Aviv University, Tel-Aviv, Israel

3 Internal Medicine Department E, Rabin Medical Center, Beilinson Hospital, Petach-Tikva, Israel

4 Internal Medicine Department C, Rabin Medical Center, Beilinson Hospital, Petach-Tikva, Israel

5 Clinical Microbiology Laboratory, Rabin Medical Center, Beilinson Hospital, Petach-Tikva, Israel

6 Internal Medicine Department A, Rabin Medical Center, Beilinson Hospital, Petach-Tikva, Israel

\section{Introduction}

Obesity is considered a global epidemic associated with an increased susceptibility to significant infections such as pneumonia and skin and soft tissue infections [1-5]. The mechanism underlying this association is not well established, but could be related to immune system dysregulation and indirectly to obesity-related co-morbidities. Moreover, it can be related to the increased leptin production which enhances the CD4 lymphocyte response towards $\mathrm{T}$ helper cell type 1 [6-8].

Scarce data have been reported on the impact of obesity on the outcome of hospitalized patients ill with infectious diseases. Various studies have found an association between obesity and poor outcome in individuals ill with the pandemic H1N1 influenza infection $[9,10]$ in addition to increased mortality among H1N1-related community acquired pneumonia (CAP) patients [11]. On the other hand, other studies have observed that obesity was independently associated with a 
reduced 30-day mortality from CAP $[12,13]$. Kahlon et al. reported low rates of in-hospital mortality in obese patients [14], whereas King et al. demonstrated that obesity may have reduced mortality in hospitalized veterans with pneumonia [15].

Influenza has been linked with increased morbidity and mortality during annual outbreaks, with a higher mortality rate during pandemic years [16-20]. Recent studies describing the changing epidemiology of influenza since the emergence of H1N1 have reported severe outcomes among obese adults. This population infected with influenza $\mathrm{A} / \mathrm{H} 1 \mathrm{~N} 1$ was more likely to require mechanical ventilation, and experience a longer LOS in an intensive care unit (ICU) and an increased risk of death [21-23]. Other epidemiological studies have shown that obesity increases the risk of severe complications and death from influenza virus infections, especially in the elderly $[24,25]$. Our aim was to examine the association between obesity (defined as a body mass index $(\mathrm{BMI}) \geq 30 \mathrm{~kg} / \mathrm{m}^{2}$ ) and adverse clinical outcomes in hospitalized adults ill with laboratory confirmed influenza during the 2017-2018 influenza season in Israel.

\section{Methods}

This is a single-center, retrospective study. Electronic records of all consecutive adult patients ( $\geq 18$ years) hospitalized between October 2017 and April 2018, diagnosed with laboratory confirmed influenza A/B virus at the Rabin Medical Center, Beilinson Hospital, Petach Tikvah, Israel (a 1000bed tertiary, university-affiliated hospital), were identified and reviewed. The collected data included baseline demographics and BMI $\left(\mathrm{kg} / \mathrm{m}^{2}\right)$ (all patients were weighed upon admission, per internal medicine ward policy). Comorbidities included the following: age-adjusted Charlson's comorbidity score, hypertension, ischemic heart disease, congestive heart disease, chronic obstructive pulmonary disease, chronic kidney disease, liver disease, malignancies, and organ transplantations. The patient encounter index upon hospital admission was as follows: vital signs, laboratory results, i.e., white blood cell count, CRP and glucose levels, chest $\mathrm{x}$-ray/CT scan results, influenza virus serotypes, vaccination status, antiviral drug therapy, invasive mechanical ventilation,vasopressors support, and ICU admission. Severe influenza complications included myocarditis or encephalitis. Patients were included once, only after the first episode.

The study cohort was divided into two groups: obesity and non-obesity. Obesity was defined as a BMI $\geq 30 \mathrm{~kg} / \mathrm{m}^{2}$. Clinical outcomes were compared between the two groups. The primary outcome was a composite of influenza-related adverse events (IRAE) including the following: 30-day allcause mortality, vasopressor support, ICU admission, mechanical ventilation, and severe influenza complications such as myocarditis or encephalitis. Secondary outcomes included the individual components of the primary outcomes, 90-day all-cause mortality, and LOS for patients discharged alive. Rehospitalization rates were measured within 90 days of the encounter index point. The study was approved by the Rabin Medical Center's Ethics Committee.

\section{Microbiology methods}

The detection method was the Simplexa ${ }^{\mathrm{TM}}$ Flu A/B \& RSV assay (https://www.focusdx.com/product/MOL2600), a realtime polymerase chain reaction (RTPCR) amplification and detection system utilizing a bi-functional fluorescent probe primer for the detection and differentiation of human influenza A virus RNA, human influenza B virus RNA, and RSV RNA on nasopharyngeal swabs performed at the Clinical Microbiology Laboratory at the Rabin Medical Center, Beilinson Hospital, Israel. The assay encompassed two main stages: (1) the extraction of RNA from patient specimens and (2) the use of a bi-functional fluorescent probe primer together with a reverse primer to amplify a specific target (for each analyte and for RNA internal control). The assay yielded three results: conserved regions of influenza A viruses (matrix gene), influenza B viruses (matrixgene), and RSV (M gene).

\section{Definitions}

Pneumonia was defined as the presence of pulmonary infiltrates observed on chest imaging, not contributable to other causes. Encephalitis was defined as a positive polymerase chain reaction (PCR) test for influenza in cerebrospinal fluid; myocarditis was defined as a positive endomyocardial biopsy for finding influenza by PCR: an influenza-like illness. Influenza-like illness (ILI) was described as a low-grade fever $\left(\geq 37.5^{\circ}\right.$ ) accompanied by at least one of the following symptoms: cough, sore throat, rhinorrhea, or myalgia. Immune deficiency was defined as a solid organ transplant, active chemotherapy for malignancy, and high-dose steroids/other immunosuppressive drugs. Hypoalbuminemia was defined as serum albumin levels $<3 \mathrm{mg} / \mathrm{dL}$.

\section{Statistical analysis}

The analysis was performed by the IBMSPSS statistics 24 software program. Statistical significance was set at a twotailed comparison with $p<0.05$. Normality distribution was assessed by the Kolmogorov-Smirnov normality and Q-Q plot test. Categorical variables were tested by the chi-square test or Fisher's exact test, as appropriate. Continuous variables were examined using Student's $t$ test if normally distributed or the Mann-Whitney $U$ test, if not. The Hosmer-Lemeshow test was used for goodness of fit. To identify individual variables associated with the composite primary outcome and a 30-day 
all-cause mortality, a univariable analysis was performed. Variables that were significantly associated with IRAE and mortality, and not highly correlated were entered into the multivariable logistic regression model. Adjusted odds ratios (ORs), 95\% confidence intervals (CIs) for IRAE, and a 30-day mortality were calculated. A subgroup analysis for the primary outcome and mortality based on BMI categories was performed: obese-BMI $30-35 \mathrm{~kg} / \mathrm{m}^{2}$; severely obese-BMI $35-40 \mathrm{~kg} / \mathrm{m}^{2}$; and morbidly obese-BMI $>40 \mathrm{~kg} / \mathrm{m}^{2}$.

\section{Results}

During the study period, 512 consecutive patients were hospitalized with laboratory-confirmed influenza A (195/512) and B (317/512). Baseline characteristics and admission data of the patients are presented in Table 1 . Obese adults were significantly younger (median age 69 (IQR 62-79) vs.76 (IQR 63-84), $p=0.013$ ), and less likely to need assistance when performing activities of daily living (ADL) compared with the control group $(10 / 83,12 \%$ vs. $115 / 417,28 \%$, $p=0.003$ ). Comorbidities were similarly distributed between the two groups. The age-adjusted Charlson's score was similar between groups (median 5 (IQR 3-6) vs. 5 (IQR 3-7); $p$ $=0.2$ ). Over half of the patients in both groups were infected with the influenza B virus $(51 / 86,60 \%$ vs. $266 / 426,62 \%$, $p=0.8$ ), and over half of the patients in both groups had received empirical antiviral therapy on admission pending microbiological confirmation $(52 / 86,61 \%$ vs. $300 / 426,70 \%$, $p=0.07)$, per hospital policy.

\section{Primary outcome}

Results are presented in Table 2. The crude rate of the composite outcome was $10 \%$ (52/507), similar between the two

Table 1 Baseline characteristics of hospitalized patients with laboratory confirmed influenza A or B infection divided into two groups (obesity group-BMI $\geq 30$ and non-obesity group $\mathrm{BMI}<30$ )

\begin{tabular}{|c|c|c|c|c|}
\hline Variable & Entire cohort $(n=512)$ & Obesity BMI $\geq 30(n=86)$ & Non-obesity BMI < $30 \quad(n=426)$ & $p$ value \\
\hline Age, median (IQR) & $74(62-83)$ & $69(62-79)$ & $76(63-84)$ & 0.01 \\
\hline Female gender, $n(\%)$ & $259(51 \%)$ & $42(49 \%)$ & $217(51 \%)$ & 0.7 \\
\hline Assistance in ADL, $n(\%)$ & $125 / 500(25 \%)$ & $10 / 83(12 \%)$ & $115 / 417(28 \%)$ & 0.003 \\
\hline Home residency, $n(\%)$ & $133 / 499(28 \%)$ & $19 / 499(27 \%)$ & $114 / 417(27 \%)$ & 0.4 \\
\hline Influenza virus type $\mathrm{B}, n(\%)$ & $317(62 \%)$ & $51(59 \%)$ & $266(62 \%)$ & 0.6 \\
\hline Influenza vaccine, $n(\%)$ & $83 / 163(51 \%)$ & $20 / 36(56 \%)$ & $63 / 127(50 \%)$ & 0.5 \\
\hline Active smoking, $n(\%)$ & $42(8 \%)$ & $10(12 \%)$ & $32(8 \%)$ & 0.2 \\
\hline Age-adjusted Charlson's score & $5(3-7)$ & $5(3-6)$ & $5(3-7)$ & 0.2 \\
\hline Hypertension, $n(\%)$ & $236(46 \%)$ & $48(56 \%)$ & $188(44 \%)$ & 0.05 \\
\hline Diabetes mellitus, $n(\%)$ & $166(32 \%)$ & $31(36 \%)$ & $135(32 \%)$ & 0.4 \\
\hline Ischemic heart disease, $n(\%)$ & $97(19 \%)$ & $22(26 \%)$ & $75(18 \%)$ & 0.08 \\
\hline Congestive heart failure, $n(\%)$ & $61(12 \%)$ & $12(14 \%)$ & $49(12 \%)$ & 0.5 \\
\hline Chronic obstructive pulmonary disease, $n(\%)$ & $56(11 \%)$ & $14(16 \%)$ & $42(10 \%)$ & 0.08 \\
\hline Chronic kidney disease, $n(\%)$ & $43(8 \%)$ & $8(9 \%)$ & $35(8 \%)$ & 0.7 \\
\hline Active malignancy, $n(\%)$ & $44(9 \%)$ & $4(5 \%)$ & $40(9 \%)$ & 0.2 \\
\hline Dementia, $n(\%)$ & $28(6 \%)$ & $5(6 \%)$ & $23(5 \%)$ & 0.9 \\
\hline Liver disease, $n(\%)$ & $6(1.2 \%)$ & $3(4 \%)$ & $3(0.7 \%)$ & 0.06 \\
\hline Organ transplant, $n(\%)$ & $22(4 \%)$ & $3(4 \%)$ & $19(5 \%)$ & 1 \\
\hline Prior statin therapy, $n(\%)$ & $183(36 \%)$ & $39(45 \%)$ & $144(34 \%)$ & 0.04 \\
\hline Temperature (Celsius) median, IQR & $37.5(36.9-38.3)$ & $37.3(36.8-38)$ & $37.5(36.9-38.3)$ & 0.4 \\
\hline Systolic blood pressure (mmHg), median (IQR) & $119(107-135)$ & $122(115-138)$ & $118(106-135)$ & 0.06 \\
\hline Saturation (\%), median (IQR) & $95(92-97)$ & $94(90-97)$ & $95(92-97)$ & 0.07 \\
\hline Pulse (beat/min), median (IQR) & $96(85-109)$ & $97(84-109)$ & $96(85-109)$ & 0.9 \\
\hline CRP level (mg/dL), median (IQR) & $5.7(2.5-13)$ & $3.2(1.2-6.6)$ & $6.1(2.7-13.2)$ & 0.005 \\
\hline Glucose level (mg/dL), median (IQR) & $137(109-181)$ & $145(118-192)$ & $138(108-180)$ & 0.2 \\
\hline WBC level (cells/cc $\left.{ }^{3}\right)$, median (IQR) & $8.34(5.8-11.5)$ & $7.1(5.8-10.4)$ & $8.4(5.8-11.9)$ & 0.4 \\
\hline Tamiflu therapy, $n(\%)$ & $352(69 \%)$ & $52(61 \%)$ & $300(70 \%)$ & 0.07 \\
\hline
\end{tabular}

$A D L$ activity of daily living, IQR interquartile range, $C R P$ C-reactive protein, $W B C$ white blood cells 
Table 2 Primary and secondary clinical outcomes in the two groups - obesity and non-obesity

\begin{tabular}{lllll}
\hline Variable & $\begin{array}{l}\text { Entire cohort } \\
(n=512)\end{array}$ & $\begin{array}{l}\text { Obesity BMI } \geq 30 \\
(n=86)\end{array}$ & $\begin{array}{l}\text { Non-obesity } \\
\text { BMI <30 }(n=426)\end{array}$ & $p$ value \\
\hline $\begin{array}{l}\text { Primary outcomes } \\
\text { Composite outcome, } n(\%)\end{array}$ & $52 / 507(10 \%)$ & $7 / 85(8 \%)$ & $45 / 422(11 \%)$ & 0.5 \\
30-day mortality, $n(\%)$ & $39(8 \%)$ & $5(6 \%)$ & $34(8 \%)$ & 0.5 \\
Vasopressors, $n(\%)$ & $17(3 \%)$ & $4(5 \%)$ & $13(3 \%)$ & 0.5 \\
Myocarditis, $n(\%)$ & $1(0.2 \%)$ & $1(1.2 \%)$ & $2(0.4 \%)$ & 0.3 \\
Encephalitis, $n(\%)$ & $2(0.5 \%)$ & $0(0 \%)$ & $2(0.4 \%)$ & 1 \\
Mechanical ventilation, $n(\%)$ & $19(4 \%)$ & $3(4 \%)$ & $16(4 \%)$ & 1 \\
ICU admission, $n(\%)$ & $20(4 \%)$ & $4(5 \%)$ & $16(4 \%)$ & 0.8 \\
Secondary outcomes & & & $4(2-7)$ & 0.5 \\
Length of hospital stay, median (IQR) & $4(2-6)$ & $3.5(2-5)$ & $101(24 \%)$ & 0.7 \\
Readmission 90 -day, $n(\%)$ & $123(24 \%)$ & $22(26 \%)$ & $51(12 \%)$ & 0.2 \\
90 -day mortality, $n(\%)$ & $57(11 \%)$ & $6(7 \%)$ & $40 / 407(10 \%)$ & 0.7 \\
Pneumonia, $n$ (\%) & $48 / 492(10 \%)$ & $8 / 85(9 \%)$ & & \\
\hline
\end{tabular}

$I C U$ intensive care unit, $I Q R$ interquartile range. groups $(7 / 85,8 \%$ vs. $45 / 422,11 \% ; p=0.5)$. The all-cause 30 day mortality rates $(5 / 86,6 \%$ vs. $34 / 426,8 \% p=0.5)$ and the 90 -day mortality rates $(6 / 86,7 \%$ vs. $51 / 426,12 \% p=0.2)$ were similar in both. Complications of myocarditis and encephalitis were rare and did not differ between groups $(1 / 86,1.2 \%$ vs. $2 / 426,0.4 \% ; p=0.3$ and $0 / 86,0 \%$ vs. $2 / 426,0.4 \% ; p=1.0$, respectively). Vasopressor use/mechanical ventilation and ICU admission were similar in both groups $(4 / 86,5 \%$ vs. $13 / 426,3 \% ; p=0.5$ and $3 / 86,4 \%$ vs. $16 / 426,4 \% ; p=14 / 86$, $5 \%$ vs. $16 / 426,4 \% ; p=0.8$, respectively). Subgroup analysis based on BMI categories revealed that the rates of the primary outcome were similar between the obese, severely obese, and morbidly obese patients $(6.6 \%(4 / 60)$ vs. $10 \%(2 / 20)$ vs. $16.6 \%(1 / 6), p=0.7$, respectively). Results for 30 - and 90 day mortality were similar between the subgroups $(6.6 \%$ $(4 / 60)$ vs. $5 \%(1 / 20)$ vs. $0 \%(0 / 6)$ and $6.6 \%(4 / 60)$ vs. $5 \%$ $(1 / 20)$ vs. $16.6 \%(1 / 6), p=0.83$ and $p=0.48$, respectively).

\section{Secondary outcomes}

LOS for patients who were discharged alive (median 3.5 days, IQR 2-5 vs. 4 days, IQR 2-7; $p=0.4$ ) and readmission rates at 90 days $(22 / 86,26 \%$ vs. $101 / 426,24 \% ; p=0.7)$ were also similar between the two groups. No significant difference in the occurrence of pneumonia was observed between the groups $(8 / 85,9 \%$ vs. $40 / 407,10 \% ; p=0.7)$. Other results are presented in Table 2.

After performing a univariable analysis, the composite outcome was found associated with increased age, low presentation systolic blood pressure/room-air saturation, hypoalbuminemia, previous stroke, chronic renal failure, and mechanical ventilation/vasopressor support/ICU admission. A multivariable model for risk factors for the primary outcome is presented in Table 3. Advanced age, chronic kidney disease, and hypoalbuminemia, not obesity, were significantly associated with the primary outcome $(\mathrm{OR}=1.03$, CI 95\% (1.0021.05); OR=2.7, CI 95\% (1.1-6.7); OR=5.4, CI 95\% (3-11); $\mathrm{OR}=1.3$, CI 95\% 0.3-3.3; $p=0.5$, respectively).

\section{Discussion}

Several significant risk factors for influenza-related adverse events were found, i.e., advanced age, chronic renal failure, and hypoalbuminemia, but not obesity. Identifying patients who are at a higher risk for influenza complications is essential, with the intention of starting early antiviral therapy and ICU admissions rather than a later transfer from a general ward. Our aim was to explore whether obesity poses a significant risk factor for adverse results, concurrently with seasonal influenza. We found that obesity exhibited no significant association with complications or mortality in adult patients

Table 3 Risk factors for composite outcome $(n=488)$

\begin{tabular}{lll}
\hline Variable & Multivariate OR (95\% CI) & $p$ value \\
\hline Age (years) & $1.03(1.002-1.05)$ & 0.03 \\
BMI $\geq 30 \mathrm{~kg} / \mathrm{m}^{2}$ & $1.3(0.5-3.3)$ & 0.5 \\
Chronic kidney disease & $2.7(1.1-6.7)$ & 0.03 \\
Flu like symptoms at presentation & $0.5(0.3-0.9)$ & 0.03 \\
Prior statin therapy & $0.7(0.3-1.4)$ & 0.3 \\
Albumin level $\leq 3 \mathrm{mg} / \mathrm{dL}$ & $5.4(3-11)$ & 0.001 \\
\hline
\end{tabular}

$B M I$ body mass index 
hospitalized in internal medicine wards due to the seasonal influenza. In addition, obesity was not associated with other clinical outcomes such as increased pneumonia rates, prolonged LOS, and increased readmission rates.

Contrary to the strong association between obesity and influenza-related morbidity and mortality reported in worldwide studies based on the $2009 \mathrm{H} 1 \mathrm{~N} 1$ pandemic data, our study suggests that obesity was not associated with adverse clinical outcomes in hospitalized adults with laboratoryconfirmed influenza A or B during the 2017-2018 influenza season. Our findings are consistent with other studies that did not find similar associations between obesity and influenza infection. In a retrospective study of $>1$ million adult patients investigated during 7 influenza seasons (2000-2007) in England, $>250,000$ obese patients experienced a decreased pneumonia rate (incidence rate ratio $=0.77$ [CI 95\% 0.680.86]) [26]. Among the risk factors for adverse events, hypoalbuminemia and chronic kidney diseases were found as risk factors for influenza-related adverse events. These findings are consistent with the findings of other studies. Studies have found that hypoalbuminemia $[27,28]$ and chronic kidney diseases [29] are associated with increased morbidity and mortality in patients with influenza.

There are several plausible explanations for our results. Firstly, the obesity group patients were younger than the controls and less likely to need assistance in ADL or reside in long-term care facilities; thus, complications and mortality might be reduced in that population. Secondly, the obesity group was more likely to use statins compared with the non-obesity group. Statin use might indirectly indicate a "healthy user effect" denoting that statin users tend to live at home, adhere to chronic medications, and engage in other positive health behaviors including adherence to an annual influenza vaccination schedule. However, the true effect of the vaccine on clinical outcomes was inestimable since data on the current influenza vaccine were unavailable for a significant number of patients. Although we were unable to obtain electronic data from the outpatient clinics, we did obtain data based on medical history alone, on the patient's current vaccination status. Thirdly, data pertaining to the time from admission to the start of antiviral therapy was sparsely reported which may have altered our results. Fourthly, the number of participating patients was not as large as planned, thus, further limiting the results. Finally, influenza B was the major circulating strain during the 2017-2018 season which might indicate a milder disease severity compared with the more severe disease from influenza A.

Our study has several advantages and strengths. This is the first study examining the association between obesity and outcomes of seasonal influenza since the last decade. Most previous studies had examined the general ambulatory population suffering from uncomplicated influenza, whereas we focused on hospitalized patients with significant comorbidities and a high rate of influenza-related adverse events.
Our study has several limitations. Firstly, this is a singlecenter experience occurring over a single influenza season; thus, the results may not be applicable at different seasons. Secondly, a further analysis of the influenza serotype was not performed. During 2017-2018, the influenza A H1N1 (pdm09) and H3N2 strains together with the influenza B strain of the Victoria lineage $(\sim 16 \%, 14 \%$, and $70 \%$ of all confirmed infections, respectively) were the most common strains circulating in the community [16]. Thirdly, due to the lack of electronic data, data relating to the current influenza vaccine were unavailable to determine the significant percentage of the patients. Instead, medical histories, including a vaccination history documenting the patient's medical records, were the only data source for vaccination status. Thus, the true effect of the vaccine on the clinical outcomes was inestimable. Finally, the time frame for the Tamiflu therapy was unavailable; hence, we could not determine whether early vs. late administration of Tamiflu impacted the outcomes of influenza in obese patients.

In conclusion, we suggest that obesity was not associated with influenza-related morbidity and mortality in hospitalized adults during the 2017-2018 influenza season. Further studies researching different influenza seasons are essential.

Acknowledgements The authors thank Mrs. Phyllis Curchack Kornspan for her editorial services.

Author contributions Jihad Bishara and Tomer Avni: study conception and design; all author: acquisition, analysis, or interpretation of data; Alaa Atamna: drafting the manuscript; all authors: critical revision of the manuscript for important intellectual content.

\section{Declarations}

Ethical approval The protocol was approved by the hospital's Research Ethics Committee.

Competing interests The authors declare no competing interests.

Consent to participate Informed consent was waived due to the noninterventional nature of the study.

\section{References}

1. Ng M, Fleming T, Robinson M, Thomson B, Graetz N, Margono C et al (2014) Global, regional, and national prevalence of overweight and obesity in children and adults during 1980-2013: a systematic analysis for the Global Burden of Disease Study 2013. Lancet 384: 766-781. https://doi.org/10.1016/S0140-6736(14)60460-8

2. Tsai AG, Wadden TA (2013) In the clinic: obesity. Ann Intern Med 159:ITC3-1-ITC3-15; quiz ITC3-16. https://doi.org/10.7326/00034819-159-5-201309030-01003

3. Falagas ME, Kompoti M (2006) Obesity and infection. Lancet Infect Dis 6:438-446. https://doi.org/10.1016/S1473-3099(06) 70523-0 
4. Farmakiotis D (2013) Obesity and serious infections. Ann Intern Med 159:859. https://doi.org/10.7326/0003-4819-159-12201312170-00021

5. Power K, Davies MM, Hargest R, Phillips S, Torkington J, Morris C (2014) A case-control study of risk factors for wound infection in a colorectal unit. Ann R Coll Surg Engl 96:37-40. https://doi.org/ 10.1308/003588414X13824511650137

6. Huttunen R, Syrjänen J (2013) Obesity and the risk and outcome of infection. Int J Obes 37:333-340. https://doi.org/10.1038/ijo.2012. 62

7. Falagas ME, Athanasoulia AP, Peppas G, Karageorgopoulos DE (2009) Effect of body mass index on the outcome of infections: a systematic review. Obes Rev 10:280-289. https://doi.org/10.1111/ j.1467-789X.2008.00546.x

8. Atamna A, Elis A, Gilady E, Gitter-Azulay L, Bishara J (2017) How obesity impacts outcomes of infectious diseases. Eur J Clin Microbiol Infect Dis 36:585-591. https://doi.org/10.1007/s10096016-2835-1

9. Kwong JC, Campitelli MA, Rosella LC (2011) Obesity and respiratory hospitalizations during influenza seasons in Ontario, Canada: a cohort study. Clin Infect Dis 53:413-421. https://doi.org/10.1093/ $\mathrm{cid} / \mathrm{cir} 442$

10. Almond MH, Edwards MR, Barclay WS, Johnston SL (2013) Obesity and susceptibility to severe outcomes following respiratory viral infection. Thorax 68:684-686. https://doi.org/10.1136/ thoraxjnl-2012-203009

11. Riquelme R, Jiménez P, Videla AJ, Lopez H, Chalmers J, Singanayagam A et al (2011) Predicting mortality in hospitalized patients with 2009 H1N1 influenza pneumonia. Int J Tuberc Lung Dis 15(4):542-546. https://doi.org/10.5588/ijtld.10.0539

12. Singanayagam A, Singanayagam A, Chalmers JD (2013) Obesity is associated with improved survival in community-acquired pneumonia. Eur Respir J 42:180-187. https://doi.org/10.1183/09031936. 00115312

13. Corrales-Medina VF, Valayam J, Serpa JA, Rueda AM, Musher DM (2011) The obesity paradox in community-acquired bacterial pneumonia. Int J Infect Dis 15:e54-e57. https://doi.org/10.1016/j. ijid.2010.09.011

14. Kahlon S, Eurich DT, Padwal RS, Malhotra A, Minhas-Sandhu JK, Marrie TJ et al (2013) Obesity and outcomes in patients hospitalized with pneumonia. Clin Microbiol Infect 19:709-716. https:// doi.org/10.1111/j.1469-0691.2012.04003.x

15. King P, Mortensen EM, Bollinger M, Restrepo MI, Copeland LA, Pugh MJ et al (2013) Impact of obesity on outcomes for patients hospitalised with pneumonia. Eur Respir J 41(4):929-934. https:// doi.org/10.1183/09031936.00185211

16. Centers for Disease Control and Prevention (2010) Estimates of deaths associated with seasonal influenza - United States, 19762007. Morb Mortal Wkly Rep 59:1057-1062

17. Hancock K, Veguilla V, Lu X, Zhong W, Butler EN, Sun H, Liu F, Dong L, DeVos JR, Gargiullo PM, Brammer TL, Cox NJ, Tumpey TM, Katz JM (2009) Cross-reactive antibody responses to the 2009 pandemic H1N1 influenza virus. NEJM 361:1945-1952. https:// doi.org/10.1056/NEJMoa0906453

18. Reichert TA, Simonsen L, Sharma A, Pardo SA, Fedson DS, Miller MA (2004) Influenza and the winter increase in mortality in the
United States, 1959-1999. Am J Epidemiol 160:492-502. https:// doi.org/10.1093/aje/kwh227

19. Simonsen L, Clarke MJ, Williamson GD, Stroup DF, Arden NH, Schonberger LB (1997) The impact of influenza epidemics onmortality:introducing a severity index. Am J Public Health 87: 1944-1950. https://doi.org/10.2105/ajph.87.12.1944

20. Atamna A, Babitch T, Bracha M, Sorek N, Haim BZ, Elis A, Bishara J, Avni T (2019) Statins and outcomes of hospitalized patients with laboratory-confirmed 2017-2018 influenza. Eur J Clin Microbiol Infect Dis 38:2341-2348. https://doi.org/10.1007/ s10096-019-03684-y

21. Morgan OW, Bramley A, Fowlkes A, Freedman DS, Taylor TH, Gargiullo P, Belay B, Jain S, Cox C, Kamimoto, Fiore A, Finelli L, Olsen SJ, Fry (2010) Morbid obesity as a risk factor for hospitalization and death due to 2009 pandemic influenza $\mathrm{A}(\mathrm{H} 1 \mathrm{~N} 1)$ disease. PLoS ONE 5:e9694. https://doi.org/10.1371/journal.pone.0009694

22. Van Kerkhove MD, Vandemaele KA, Shinde V, JaramilloGutierrez G, Koukounari A, Donnelly CA et al (2011) Risk factors for severe outcomes following 2009 influenza A (H1N1) infection: a global pooled analysis. PLoS Med 8:e1001053. https://doi.org/ 10.1371/journal.pmed.1001053samerefas 25

23. Martin ET, Archer C, McRoberts J, Kulik J, Thurston T, Lephart P, Kaye KS (2013) Epidemiology of severe influenza outcomes among adult patients with obesity in Detroit, Michigan, 2011. Influenza Other Respir Viruses 7:1004-1007. https://doi.org/10. 1111/irv.12115

24. Centers for Disease Control and Prevention (CDC) (2009) Intensive-care patients with severe novel influenza A (H1N1) virus infection-Michigan, June 2009. Morb Mortal Wkly Rep 58:749752

25. Maier HE, Lopez R, Sanchez N, Ng S, Gresh L, Ojeda S et al (2018) Obesity increases the duration of influenza A virua shedding in adults. J Infect Dis 218:1378-1382. https://oi.org/10.1093/ infdis/jiy370

26. Blumentals WA, Nevitt A, Peng MM, Toovey S (2012) Body mass index and the incidence of influenza-associated pneumonia in a UK primary care cohort. Influenza Other Respir Viruses 6:28-36. https://doi.org/10.1111/j.1750-2659.2011.00262.x

27. Zhang ZXZ, Mar Kyaw W, Ho HJ, Tay MZ, Huang H, Aung Hein A, Chow A (2011) Seasonal influenza-associated intensive care unit admission and death in tropical Singapore, 2011-2015. J Clin Virol 117:73-79. https://doi.org/10.1016/j.jcv.2019.06.005

28. Avni T, Babich T, Nir A, Yahav D, Shaked H, Sorek N, Zvi HB, Bishara J, Atamna A (2020) Comparison of clinical outcomes of influenza A and B at the 2017-2018 influenza season: a cohort study. Eur J Clin Microbiol Infect Dis 39:1109-1114. https://doi. org/10.1007/s10096-020-03822-x

29. Reddy S, Chitturi C, Yee J (2019) Vaccination in chronic kidney disease. Adv Chronic Kidney Dis 26:72-78. https://doi.org/10. 1053/j.ackd.2018.10.002

Publisher's note Springer Nature remains neutral with regard to jurisdictional claims in published maps and institutional affiliations. 